\title{
On the Generic Robustness of Solution Concepts to Incomplete Information*
}

\author{
Guilherme Carmona ${ }^{\dagger}$ \\ University of Surrey
}

December 11, 2017

\begin{abstract}
We consider the generic robustness of an upper hemi-continuous solution concept on a class of games of interest which has been embedded in a larger space of games. We show that generic robustness follows if the class of games of interest is "large" relative to the class of games in which it has been embedded. This result is used to show why interim correlated rationalizable actions are generically robust to players' hierarchies of beliefs as established by Weinstein and Yildiz (2007) even without their richness condition. It is also used to provide a formal sense according to which, in the setting of Kajii and Morris (1997b), the set of representations of a complete information game is small in the space of incomplete information games in which it is embedded. This difference in relative sizes makes the robustness problems of Kajii and Morris (1997b) and Weinstein and Yildiz (2007) be incomparable and helps explaining why their conclusions differ so significantly.
\end{abstract}

*I wish to thank Christian Hellwig, Atsushi Kajii, Mário Páscoa, Satoru Takahashi and two anonymous referees for very helpful comments. Special thanks to Siyang Xiong for very detailed comments. Any remaining errors are, of course, mine.

${ }^{\dagger}$ Address: University of Surrey, School of Economics, Guildford, GU2 7XH, UK; phone: (44) 148368 3475; email: g.carmona@surrey.ac.uk. 


\section{Introduction}

Robustness problems abound in game theory. Amongst those robustness problems, the one concerning the robustness of Nash equilibrium of complete information games to incomplete information has received considerable attention. Specifically, Kajii and Morris (1997b), and Carlsson and van Damme (1993) before, have shown that some Nash equilibria of some normal-form games are not robust to incomplete information. This lack of robustness of Nash equilibrium of complete information games to incomplete information is particularly striking because it holds for some strict Nash equilibria, which were thought to be robust to all sorts of perturbations. Furthermore, the lack of robustness of Nash equilibrium to incomplete information is robust in the sense that it holds on an open subset of complete information games.

A quite different message arises from the robustness problem considered in Weinstein and Yildiz (2007). In contrast to the above robust lack of robustness of Nash equilibrium to incomplete information, Weinstein and Yildiz (2007) have shown that interim correlated rationalizable actions are generically robust to players' beliefs. The relevance of this result to those of Kajii and Morris (1997b) is that, while the entire hierarchy of players' beliefs is not explicitly part of the notion of robustness used by Kajii and Morris (1997b), players' beliefs are central to their analysis and results. Thus, Weinstein and Yildiz's (2007) paper is a natural continuation of Kajii and Morris's (1997b) work of analyzing the robustness of equilibria to incomplete information. It is then surprising that Weinstein and Yildiz (2007) obtained a conclusion that differs so significantly from the one reached in Kajii and Morris (1997b). ${ }^{1}$ Why is this?

In this paper, we provide a unified view on robustness problems. Specifically, we define a robustness problem by a class of games of interest, a (topological) space of games in which the former is embedded (the perturbation space, for short), a

\footnotetext{
${ }^{1}$ Different results arise when one focus on full (as opposed to generic) robustness. See Kajii and Morris (1997a) and the references therein, and also Weinstein and Yildiz (2007), namely their structure theorem (Proposition 1 in their paper). See also Ely and Pȩski (2011), Chen (2012), Penta (2012) and Penta (2013) amongst others for further results.
} 
(metric) space where the solutions lie and a solution concept described by a (nonempty and compact-valued, upper hemi-continuous) correspondence from the perturbation space to the solution space. Using Fort's (1951) theorem on the generic continuity of correspondences, we then show that if class of games of interest is non-meager in the perturbation space, then the solution concept is continuous on a non-meager subset of the class of games of interest. Moreover, if class of games of interest is dense in the perturbation space, then the solution concept is continuous on a residual subset of the class of games of interest. Our notion of a generic set in a topological space is that of a residual set and, thus, we interpret the latter result as stating that the solution concept is generically robust provided that the class of games of interest is sufficiently large relative to the perturbation space. ${ }^{2}$

The above result reveals that the generic robustness result of Weinstein and Yildiz (2007) holds because they focus on a class of games of interest that is large in the space of perturbations. Indeed, this robustness problem is one where the perturbation space consists of the universal type space of Mertens and Zamir (1985). The set of games (i.e. types) of interest is any dense subset of the universal type space and, therefore, the generic robustness of interim correlated rationalizable actions to hierarchies of players' beliefs follows from our abstract result. In fact, by exploring the finiteness of the strategy space, we show that the correspondence of interim correlated rationalizable action profiles is locally constant in an open and dense subset of any dense subset of the universal type space, thus extending an analogous result of Weinstein and Yildiz (2007) by dispensing with their richness assumption. ${ }^{3}$

The (contrapositive of the) above result also shown that, in the setting of Kajii

\footnotetext{
${ }^{2}$ Chen and Xiong (2013) also refer to residual sets as generic sets. When the perturbation space is a Baire space, then each of its residual subsets can be interpreted as being not small and as having a small complement. See Section 2 for details.

${ }^{3}$ It is interesting to note that this result depends only on the upper hemi-continuity of the correspondence of interim correlated rationalizable action profiles and not on deeper properties of this correspondence and of the universal type space. By exploring these deeper properties, Weinstein and Yildiz (2007) obtained conclusions that are stronger than the generic robustness of the correspondence of interim correlated rationalizable action profiles; see below.
} 
and Morris (1997b), the set of representations of a complete information game is small (meager and not dense) in the space of incomplete information games in which the former set has been embedded. This is so not just at an intuitive level, but also formally in the following sense: If the robustness problem is formalized such that the solution correspondence is upper hemi-continuous and lower hemi-continuity of the solution correspondence corresponds to Kajii and Morris's (1997b) notion of robustness to incomplete information, then the set of games of interest must indeed be small in the perturbation space whenever some Nash equilibrium of the given complete information game fails to be robust to incomplete information. We show that a formalization of the Kajii and Morris's (1997b) robustness problem that have these properties exists.

The above consequences of our main result mean that the reason why the generic robustness result of Weinstein and Yildiz (2007) and the robust lack of robustness result of Kajii and Morris (1997b) differ so significantly lies, in part, on the difference in the size of the class of games of interest relative to the perturbation space in their robustness problems. In particular, our results provide a sense in which the robustness of a solution concept is independent of the actual space where this robustness exercise is performed, namely whether the perturbation space is a space of priors as in Kajii and Morris (1997b) or a space of hierarchies of beliefs as in Weinstein and Yildiz (2007), but rather depends on the topological properties of the class of games of interest as a subset of the perturbation space.

We emphasize that the focus of this paper is on the generic robustness of solution concepts and, in particular, on the implications of the results in Kajii and Morris (1997b) and Weinstein and Yildiz (2007) for this issue. It is worth mentioning that Kajii and Morris (1997b) went well beyond showing that some Nash equilibria of some normal-form games are not robust to incomplete information, namely, by obtaining a classification of which games of complete information are robust to incomplete information. Likewise, Weinstein and Yildiz (2007) went well beyond establishing the generic robustness of interim correlated rationalizable actions. In fact, they obtained the generic uniqueness of interim correlated rationalizable actions from which generic 
robustness follows. In fact, they have shown that, for each possible action profile, each point of multiplicity is the limit point of a sequence of points have that action profile as a unique solution. As already suggested in Footnote 1, our paper has nothing to say on these results.

The paper is organized as follows. In Section 2, we present our setting and our result on generic robustness. This result is then applied in Sections 2.1 and 2.2 to the robustness problems of Kajii and Morris (1997b) and Weinstein and Yildiz (2007). Some concluding remarks, including open questions motivated by the above results are in Section 3. The proofs of our results are in the Appendix.

\section{Generic robustness}

In this section, we present the notion of a generic set that we use and our result on generic robustness in an abstract setting. This setting is then specialized in Sections 2.1 and 2.2 .

Let $X$ be a topological space. A subset $T$ of $X$ is nowhere dense in $X$ if $\operatorname{int}(\operatorname{cl}(T))=\emptyset$ and it is meager in $X$ if it is a countable union of nowhere dense sets. ${ }^{4}$ A set $T \subseteq X$ is non-meager in $X$ if it is not meager in $X$ and is residual in $X$ if it is the complement of a meager set in $X$. The space $X$ is said to be a Baire space if every nonempty open set in $X$ is non-meager. ${ }^{5}$ Note that each residual set in a nonempty Baire space $X$ is non-meager since, otherwise, $X$ would be a nonempty open meager set. Hence, intuitively, a residual set in a nonempty Baire space has a "small" complement and is itself not "small". For these reasons, whenever $A \subseteq X$ is residual in $X$, we also say that $A$ is generic in $X$ (in this case, we also say that $A$ is a generic subset of $X$ ).

With the above notion of a generic set, we proceed to the notion of generic ro-

\footnotetext{
${ }^{4}$ Given a subset $A$ of a topological space, $\operatorname{int}(A)$ denotes the interior of $A, \operatorname{cl}(A)$ the closure of $A$ and $A^{c}$ the complement of $A$.

${ }^{5}$ See Bourbaki (1966, p. 192) for several equivalent definitions of a Baire space. Recall that Baire's category theorem assets that every complete pseudo-metric space is a Baire space (see e.g. Kelley (1955, Theorem 34, p. 200)).
} 
bustness. A robustness problem is described by a space of games $X$, assumed to be a topological space, a class of games in $X$ described by a subset $A$ of $X$, a solution space $S$, assumed to be a metric space, and a solution concept described by a correspondence $\Psi: X \rightrightarrows S$, where $\Psi$ is assumed to be upper hemi-continuous with nonempty and compact values. The notion of a robustness problem covers situation where one is interested in a class $A$ of games which is embedded in a larger space $X$, interpreted as a space of perturbations, and the solution correspondence $\Psi$ is upper hemi-continuous with nonempty and compact values (the typical case in many applications). The robustness issue is whether or not $\Psi$ is also lower hemi-continuous. We then say that (the solution concept described by) $\Psi$ is generically robust on $A$ relative to $X$ if $\Psi$ is continuous on a generic subset of $A{ }^{6}$

We also use the following notion of robustness: $\Psi$ is weakly robust on $A$ relative to $X$ if $\Psi$ is continuous on a non-meager subset of $A$. Note that, whenever $A$ (endowed with the relative topology induced by the topology of $X$ ) is a Baire space, weak robustness on $A$ relative to $X$ is indeed a weaker notion than generic robustness on $A$ relative to $X$. While we require neither $X$ nor $A$ to be a Baire space in the formal definition of a robustness problem, $X$ is, in fact, a Baire space in all the robustness problems we consider, the same holding regarding $A$ except possibly in the robustness problem of Corollary 2 below when $A$ is merely a dense subset of $X$. Hence, as discussed above, generic sets are non-meager in these spaces.

The following result provides sufficient conditions on $A$ for $\Psi$ to be weakly and generic robust on $A$ relative to $X$. It is largely a consequence of Fort's (1951) theorem on the generic continuity of correspondences. ${ }^{7}$

Theorem 1 Let $(X, A, S, \Psi)$ be a robustness problem.

\footnotetext{
${ }^{6}$ Note that the space $X$ matter for this definition because it is the domain of $\Psi$. In fact, a quite different notion of generic robustness would result if only the restriction of $\Psi$ to $A$ was required to be continuous.

${ }^{7}$ Fort's (1951) theorem is widely used to address the essentiality of Nash equilibrium in discontinuous games. See, e.g. Carbonell-Nicolau (2010) and Scalzo (2013); see also Carmona (2013, Chapter 5) for a survey of this literature.
} 
1. If $A$ is non-meager in $X$, then $\Psi$ is weakly robust on $A$ relative to $X$.

2. If $A$ is dense in $X$, then $\Psi$ is generically robust on $A$ relative to $X$.

3. If $S$ is finite and $A$ is dense (resp. non-meager) in $X$, then there exists an open and dense (resp. non-meager) subset $O$ of $A$ such that $\Psi$ is locally constant on 0.8

Theorem 1 implies that the solution concept $\Psi$ in a robustness problems is (generically or weakly) robust provided that the class $A$ of games of interest is sufficiently large in the space $X$ of perturbations. For weak robustness, it suffices that $A$ be non-meager in $X$ and, for generic robustness, that $A$ be dense in $X$ (which holds, in particular, if $X$ is a Baire space and $A$ is generic in $X$ ). Furthermore, a stronger conclusion is obtained in the case where the solution space is finite, namely that the solution correspondence is locally constant in an open subset of the class $A$ of games of interest; this open set is dense in $A$ if $A$ is dense in $X$ and non-meager in $A$ if $A$ is non-meager in $X$.

\subsection{Robustness to incomplete information}

In this section, we consider the robustness of the Nash equilibria of a given complete information game to incomplete information as in Kajii and Morris (1997b).

A finite normal-form game $G=\left(C_{i}, g_{i}\right)_{i \in N}$ consists of a finite set $N$ of players and, for each player $i \in N$, a nonempty, finite pure-strategy space $C_{i}$ and a payoff function $g_{i}: C \rightarrow \mathbb{R}$, where $C=\prod_{i \in N} C_{i}$. Let, for each finite set $B, \Delta(B)=\{y \in$ $\left.\mathbb{R}_{+}^{|B|}: \sum_{b \in B} y_{b}=1\right\}$ and $S_{i}=\Delta\left(C_{i}\right)$ denote the set of player $i$ 's mixed strategies for each $i \in N$. Throughout the paper, subsets of Euclidean spaces are endowed with the sup norm, i.e. $\|x\|=\sup _{k=1, \ldots, m}\left|x_{k}\right|$ whenever $x \in \mathbb{R}^{m}$. Define $S=\prod_{i \in N} S_{i}$, $s(c)=\prod_{i \in N} s_{i}\left(c_{i}\right)$ and $g_{i}(s)=\sum_{c \in C} s(c) g_{i}(c)$ for each $i \in N, c \in C$ and $s \in S$, where $s_{i}\left(c_{i}\right)$ denotes the probability that $c_{i}$ is played under $s_{i}$. The symbol $-i$ denotes "all

\footnotetext{
${ }^{8}$ I.e. for each $x \in O$, there exists an open neighborhood $U$ of $x$ in $A$ such that $\Psi\left(x^{\prime}\right)=\Psi(x)$ for each $x^{\prime} \in U$.
} 
players but $i "$ and $S_{-i}=\prod_{j \neq i} S_{j}$ denotes the set of mixed strategy profiles for all players but $i$. A Nash equilibrium of $G$ is $s^{*} \in S$ such that $g_{i}\left(s^{*}\right) \geq g_{i}\left(s_{i}, s_{-i}^{*}\right)$ for all $i \in N$ and $s_{i} \in S_{i}$.

Let $G=\left(C_{i}, g_{i}\right)_{i \in N}$ be a fixed finite normal-form game. The game $G$ is embedded in a space of incomplete information games as follows. Each incomplete information game in this space is defined by the fixed player set $N$, the fixed action sets $C_{1}, \ldots, C_{n}$, a countable state space $\Omega$, a probability measure $P$ on $\Omega$ and, for each $i \in N$, a bounded state dependent payoff function $u_{i}: C \times \Omega \rightarrow \mathbb{R}$ and a partition $\mathcal{Q}_{i}$ of $\Omega$ such that $P\left(Q_{i}(\omega)\right)>0$ for all $\omega \in \Omega$, where $Q_{i}(\omega)$ is the unique element of $\mathcal{Q}_{i}$ containing $\omega$. Let $E(G)$ be the set of all such incomplete information games whose payoff functions are bounded by a common constant.

Let $U=\left(\Omega, P,\left(C_{i}, u_{i}, \mathcal{Q}_{i}\right)_{i \in N}\right) \in E(G)$. A mixed strategy for player $i \in N$ is a $\mathcal{Q}_{i}$-measurable function $\sigma_{i}: \Omega \rightarrow \Delta\left(C_{i}\right)$. Let $\sigma_{i}\left(c_{i} \mid \omega\right)$ denote the probability that $c_{i}$ is played given $\omega$ under $\sigma_{i}$ for each $i \in N, c_{i} \in C_{i}$ and $\omega \in \Omega$. Furthermore, let $\sigma=\left(\sigma_{1}, \ldots, \sigma_{n}\right), \sigma(c \mid \omega)=\prod_{i \in N} \sigma_{i}\left(c_{i} \mid \omega\right)$ and $u_{i}(\sigma(\omega), \omega)=\sum_{c \in C} \sigma(c \mid \omega) u_{i}(c, \omega)$ for each $i \in N, c \in C$ and $\omega \in \Omega$. Let $P\left(\omega \mid Q_{i}(\omega)\right)=P(\omega) / P\left(Q_{i}(\omega)\right)$ be the conditional probability of state $\omega$ given information set $Q_{i}(\omega)$ for each $i \in N$ and $\omega \in \Omega$. We say that $\sigma$ is a Bayesian Nash equilibrium of $U$ if

$$
\sum_{\omega^{\prime} \in Q_{i}(\omega)} P\left(\omega^{\prime} \mid Q_{i}(\omega)\right) u_{i}\left(\sigma\left(\omega^{\prime}\right), \omega^{\prime}\right) \geq \sum_{\omega^{\prime} \in Q_{i}(\omega)} P\left(\omega^{\prime} \mid Q_{i}(\omega)\right) u_{i}\left(\left(c_{i}, \sigma_{-i}\left(\omega^{\prime}\right)\right), \omega^{\prime}\right)
$$

for all $i \in N, c_{i} \in C_{i}$ and $\omega \in \Omega$. Furthermore, an equilibrium action distribution of $U$ is $\mu \in \Delta(C)$ such that, for some Bayesian Nash equilibrium $\sigma$ of $U, \mu(c)=$ $\sum_{\omega \in \Omega} \sigma(c \mid \omega) P(\omega)$ for each $c \in C$. We say that $\mu \in \Delta(C)$ is an Nash equilibrium action distribution of $G$ if, for some Nash equilibrium $\sigma$ of $G, \mu(c)=\sigma(c)$ for each $c \in C$ (i.e. $\left.\mu(c)=\prod_{i \in N} \sigma_{i}\left(c_{i}\right)\right)$.

The notion of robustness of a Nash equilibrium of $G$ to incomplete information is defined as follows. For each $U=\left(\Omega, P,\left(C_{i}, u_{i}, \mathcal{Q}_{i}\right)_{i \in N}\right) \in E(G)$, let

$$
\Omega_{U}=\left\{\omega \in \Omega: u_{i}\left(c, \omega^{\prime}\right)=g_{i}(c) \text { for all } c \in C, \omega^{\prime} \in Q_{i}(\omega) \text { and } i \in N\right\} \text {. }
$$

For each $\varepsilon \in[0,1]$, we say that $U \in E(G)$ is an $\varepsilon$-elaboration of $G$ if $P\left(\Omega_{U}\right)=1-\varepsilon$ and let $E(G, \varepsilon)$ be the set of all $\varepsilon$-elaborations of $G$. Finally, we say that an action 
distribution $\mu \in \Delta(C)$ is robust to incomplete information in $G$ if, for every $\delta>0$, there exists $\bar{\varepsilon}>0$ such that, for all $0 \leq \varepsilon \leq \bar{\varepsilon}$, every $U \in E(G, \varepsilon)$ has an equilibrium action distribution $\nu$ such that $\|\mu-\nu\| \leq \delta$.

The above notion of robustness to incomplete information is now expressed in terms of a robustness problems as defined in Section 2. The space $X$ of games is $E(G), S$ is the (metric) space $\Delta(C)$ of action distributions, and $A$ is the set of 0 elaborations of $G$, i.e. $A=E(G, 0)$.

We want to specify the remaining elements, the solution correspondence $\Psi$ and the topology $\tau$ on $X$, such that $(X, A, S, \Psi)$ is a robustness problem that reflects Kajii and Morris's (1997b) notion of robustness to incomplete information, in the sense that the lower hemi-continuity of $\Psi$ at some $U \in A$ implies that all Nash equilibrium action distributions of $G$ are robust to incomplete information. Theorem 2 asserts that this can be done and in way that (a) $A$ is meager and non-dense in $X$ (meaning that the set of representations of the complete information game $G$ as 0-elaborations of $G$ is small in the space of incomplete information games $E(G)$ ) and (b) both $X$ and $A$ are Baire spaces (meaning that residual sets are non-meager).

Theorem 2 There is a correspondence $\Psi: X \rightrightarrows S$ and a topology $\tau$ on $X$ such that

1. (X, A, S, $\Psi)$ is a robustness problem that reflects Kajii and Morris's (1997b) notion of robustness to incomplete information,

2. A is closed and nowhere dense in $X$, and

3. Both $X$ (endowed with $\tau$ ) and $A$ (endowed with its relative topology induced by $\tau)$ are Baire spaces.

Theorem 2 shows that it is possible to formulate a robustness problem that reflects Kajii and Morris's (1997b) notion of robustness to incomplete information in which $A$ is small relative to $X$, i.e. $A$ is both meager and non-dense in $X$. The main point of this section is that, when game $G$ is such that not all of its Nash equilibrium action distributions are robust to incomplete information, $A$ is necessarily small relative 
to $X$ in any robustness problem that reflects Kajii and Morris's (1997b) notion of robustness to incomplete information. ${ }^{9}$

Corollary 1 Let $G$ be such that not all of its Nash equilibrium action distributions are robust to incomplete information, $\tau^{\prime}$ be a topology on $X$ and $\Psi^{\prime}: X \rightrightarrows S$ be a correspondence.

If, when $X$ is endowed with $\tau^{\prime},\left(X, A, S, \Psi^{\prime}\right)$ is a robustness problem that reflects Kajii and Morris's (1997b) notion of robustness to incomplete information, then $A$ is a meager and non-dense subset of $X$.

Corollary 1 is a simple consequence of Theorem 1. Indeed, because $\left(X, A, S, \Psi^{\prime}\right)$ reflects Kajii and Morris's (1997b) notion of robustness to incomplete information, it follows that $\Psi$ is not continuous at $U$ for all $U \in A$. Hence, $\Psi$ is neither weakly nor generically robust on $A$ relative to $X$. By Theorem 1 , this can occur only when $A$ is a meager and non-dense subset of $X$, i.e. a game $G$ is meager and non-dense (and, hence, non-generic) in the space of all incomplete information games in which it is embedded.

In the context of this section, one may want to test the robustness to incomplete information of the solution, described by $\Psi$, of games that may be of incomplete information. That this can be done is an easy consequence of Theorem 1 . Indeed, if $(X, A, S, \Psi)$ is a robustness problem, $A$ is meager in $X$ and $X$ is a Baire space, then $\Psi$ is generically robust on $X$ and on $A^{c}$, both relative to $X$ (this follows because both $X$ and $A^{c}$ are dense in $X$ ). In addition, this conclusion can be reached even when one wishes to consider a solution concept $\Phi$ which is not necessarily upper hemi-continuous at points of $A$; for instance, this happens when, in the context of this session, $\Phi$ is the Bayesian Nash equilibrium correspondence (see the proof of Theorem 2).

\footnotetext{
${ }^{9}$ In particular, this means that the conclusion that $A$ is meager and non-dense in $X$ that follows from part 2 of Theorem 2 does not depend on the particular choices for $\Psi$ and $\tau$ we made in its proof.
} 
Remark 1 If $(X, A, S, \Psi)$ is a robustness problem, $A$ is closed and meager in $X$, and $\Phi: X \rightrightarrows S$ is such that $\Phi(x) \subseteq \Psi(x)$ for each $x \in X$ and $\Phi(x)=\Psi(x)$ for each $x \in A^{c}$, then $\Phi$ is generically robust on $X$ and on $A^{c}$, both relative to $X$.

The proof of Theorem 2 shows that $\Psi$ and the topology $\tau$ on $X$ can be chosen such that all of the assumptions of Remark 1 are satisfied (and in a way that the robustness problem reflects Kajii and Morris's (1997b) notion of robustness to incomplete information) when $\Phi$ is the correspondence of the (Bayesian Nash) equilibrium action distributions. More generally, for any such choice of $\Psi$ and $\tau$ such that all of the assumptions of Remark 1 hold, Remark 1 then implies that Bayesian Nash equilibrium of incomplete information games is generically robust to incomplete information.

\subsection{Robustness to hierarchies of beliefs}

We next consider the problem of robustness of interim correlated rationalizable actions to hierarchies of players' beliefs following Weinstein and Yildiz (2007). The setting is described by a finite set $N$ of players, a parameter space $\Theta^{*}$, assumed to be a compact metric space, and, for each $i \in N$, a finite action space $C_{i}$ and a continuous payoff function $u_{i}: \Theta^{*} \times C \rightarrow \mathbb{R}$. We consider the set of games that differ in their specification of the belief structure on $\theta$, here described by different types in the universal type space of Mertens and Zamir (1985). Thus, in this setting, the type space of each player $i \in N$ is the universal type space $T_{i}^{*}$ consisting of all belief hierarchies for which it is common knowledge that the beliefs are coherent.

A brief description of the universal type space $T_{i}^{*}$ of player $i \in N$ is as follows; see Mertens and Zamir (1985) and Brandenburger and Dekel (1993) for details. Due to the underlying uncertainty reflected in $\Theta^{*}$, each player has beliefs over $\Theta^{*}$ (his firstorder beliefs), beliefs over the beliefs of other players (second-order beliefs) and so on. Each type $t_{i} \in T_{i}^{*}$ consists of one such hierarchy of beliefs. For each compact metric space $K$, let $M(K)$ denote the space of Borel probability measures on $K$ endowed with the narrow topology, ${ }^{10}$ and define the sets $\left(Y_{0}, Y_{1}, \ldots\right)$ and $\left(M_{1}, M_{2}, \ldots\right)$ by $Y_{0}=\Theta^{*}$,

\footnotetext{
${ }^{10}$ Recall that the narrow topology on $M(X)$ is defined to be the smallest topology on $M(K)$
} 
$M_{k}=M\left(Y_{k-1}\right)$ and $Y_{k}=Y_{k-1} \times\left(M_{k}\right)^{n}=\Theta^{*} \times \prod_{l=1}^{k}\left(M_{l}\right)^{n}$ for all $k \in \mathbb{N}$. We then have that the universal type space $T_{i}^{*}$ of player $i$ is a subset of $\prod_{k=0}^{\infty} M\left(Y_{k}\right)$, which is endowed with the relative topology induced from the product topology of $\prod_{k=0}^{\infty} M\left(Y_{k}\right)$. Let $T^{*}=\prod_{i \in N} T_{i}^{*}$. For each $i \in N$ and $t_{i} \in T_{i}^{*}$, let $\kappa_{t_{i}} \in M\left(\Theta^{*} \times T_{-i}^{*}\right)$ be the unique probability measure that represents the beliefs of $t_{i}$ about $\left(\theta, t_{-i}\right)$.

The set of interim correlated rationalizable actions for each type $t_{i} \in T_{i}^{*}$ of player $i \in N$ is defined as follows. Let $S_{i}^{0}\left(t_{i}\right)=C_{i}$ for all $i \in N$ and $t_{i} \in T_{i}^{*}$ and, for each $k \in \mathbb{N}$, assume that $S_{i}^{1}\left(t_{i}\right), \ldots, S_{i}^{k-1}\left(t_{i}\right)$ have been defined for all $i \in N$ and $t_{i} \in T_{i}^{*}$. For each $i \in N$ and $t_{i} \in T_{i}^{*}$, let $S_{-i}^{k-1}\left(t_{-i}\right)=\prod_{j \neq i} S_{j}^{k-1}\left(t_{j}\right)$ and define $S_{i}^{k}\left(t_{i}\right)$ to be the set of $c_{i} \in C_{i}$ with the property that there exists $\pi \in M\left(\Theta^{*} \times T_{-i}^{*} \times C_{-i}\right)$ such that (a) the marginal of $\pi$ on $\Theta^{*} \times T_{-i}^{*}$ is $\kappa_{t_{i}}$, (b) $\pi\left(\left\{\left(\theta, t_{-i}, c_{-i}\right) \in \Theta^{*} \times T_{-i}^{*} \times C_{-i}: c_{-i} \in\right.\right.$ $\left.\left.S_{-i}^{k-1}\left(t_{-i}\right)\right\}\right)=1$ and $(\mathrm{c}) c_{i}$ solves

$$
\max _{c_{i}^{\prime} \in C_{i}} \int_{\Theta^{*} \times C_{-i}} u_{i}\left(\theta, c_{i}^{\prime}, c_{-i}\right) \mathrm{d} \pi^{\prime}\left(\theta, c_{-i}\right),
$$

where $\pi^{\prime}$ is the marginal of $\pi$ on $\Theta^{*} \times C_{-i}$. The set of interim correlated rationalizable actions for type $t_{i} \in T_{i}^{*}$ of player $i \in N$ is $S_{i}^{\infty}\left(t_{i}\right)=\bigcap_{k=0}^{\infty} S_{i}^{k}\left(t_{i}\right)$. Let, for each $t \in T^{*}$, $S^{\infty}(t)=\prod_{i \in N} S_{i}^{\infty}\left(t_{i}\right)$.

We define a robustness problem by letting $X=T^{*}, A$ be a dense subset in $T^{*}$, $S=C$ and $\Psi(t)=S^{\infty}(t)$ for each $t \in T^{*}$. Since $X$ is a topological space (and also a Baire space since it is a compact metric space by Mertens and Zamir (1985, Theorem 2.9)), $S$ is a metric space and $\Psi$ is upper hemi-continuous with nonempty and compact values (see Weinstein and Yildiz (2017, Proposition 1)), it then follows from Theorem 1 that $\Psi$ is generically robust on $A$ relative to $X$ and, in fact, locally constant in an open and dense subset of $A$.

Corollary 2 Let $X, S$ and $\Psi$ be as defined above and $A$ be a dense subset of $X$. Then there exists an open and dense subset $O$ of $A$ such that $\Psi$ is locally constant on $O$.

for which all functions of the form $\mu \mapsto \int_{K} f \mathrm{~d} \mu$ are continuous, where $f: K \rightarrow \mathbb{R}$ is bounded and continuous (see Fremlin $(2003,437 \mathrm{~J}$, p. 229)). It is also known as the topology of the weak convergence of probability measures and as the weak* topology. 
Corollary 2 states that interim correlated rationalizable actions are generically robust in a strong sense in the universal type space relative to itself and, also, in any dense subset of the universal type space relative to the universal type space. ${ }^{11}$ It reaches a conclusion analogous to those of Proposition 2 and Corollary 1 in Weinstein and Yildiz (2007), which also asserted that $\Psi$ is locally constant in an open and dense subset of $A$ for any dense subset $A$ of $X$, while dispensing with their richness assumption (Assumption 1 in their paper). However, without this richness assumption, we do not obtain the conclusion in Proposition 2 and Corollary 1 in Weinstein and Yildiz (2007) that $\Psi$ is locally unique. ${ }^{12,13}$

The above conclusion contrasts with the one obtained in Section 2.1. While there are many differences between the respective robustness problems, an important one concerns the set $A$ of games. Indeed, in Section 2.1, $A$ is meager and non-dense in the perturbation space $X$ in which $A$ is embedded, whereas in Corollary 2 above, $A$ is a dense subset of $X$. This difference is important because were $A$ dense in $X$ in the robustness problem of Section 2.1, then $\Psi$ would then be generically robust on $A$ relative to $X$. This difference makes the robustness problems in Sections 2.1 and 2.2 be incomparable as far as the generic robustness of the solution concept is concerned and helps explaining why their conclusions differ significantly.

\footnotetext{
${ }^{11}$ Two dense subsets of the universal type space that are of special interest are the set of finite types and the set of finite types consistent with the common-prior assumption. See Weinstein and Yildiz (2007) for details; the denseness of each of these two sets was originally established by Mertens and Zamir (1985) and Lipman (2003) respectively.

${ }^{12}$ Corollary 2 with $A=X=T^{*}$ implies Theorem 3 in Ely and Pesski (2011), which roughly states that the set of "regular" types contain an open and dense set. Indeed, it is easy to see that if $t_{i} \in T_{i}^{*}$ is such that $S_{i}^{\infty}\left(t_{i}\right)$ is locally constant, then $t_{i}$ is regular.

${ }^{13}$ In the terminology of Chen, Takahashi, and Xiong (2014), Corollary 2 states that, for each $t$ in a open dense subset of $T^{*}, S_{i}^{\infty}\left(t_{i}\right)$ (and, in fact, any nonempty subset of it) is a robust prediction for type $t_{i}$ of player $i \in N$. See their paper for details and for a characterization of fully (as opposed to generically) robust predictions.
} 


\section{Concluding remarks}

We have provided a unified view on several robustness problems by defining an abstract notion of a robustness problem. We then showed that the generic robustness of the solution concept follows if the class of games of interest is dense in the space of games in which it is embedded. This result implies that the interim correlated rationalizable action profiles are generically robust to players' hierarchies of beliefs in the setting of Weinstein and Yildiz (2007).

We have also shown that, in the setting of Kajii and Morris (1997b), the set of representations of a complete information game is small (meager and not dense) in the space of incomplete information games in which the former set has been embedded. This result and the one above together mean that the generic robustness result of Weinstein and Yildiz (2007) and the robust lack of robustness result of Kajii and Morris (1997b) are incomparable, and help explaining why their conclusions differ so significantly.

The robustness problem of Kajii and Morris (1997b) can be made comparable to that of Weinstein and Yildiz (2007) by taking in the latter the space of incomplete information games to be the class of games of interest. In this case, the class of games of interest is large relative to the perturbation space and thus we obtained the generic robustness of Bayesian Nash equilibrium to incomplete information. Thus, when the two results are made comparable, their conclusion are analogous.

To strengthen the above view, it would be interesting to consider a robustness problem analogous to the one of Kajii and Morris (1997b) in the setting of Weinstein and Yildiz (2007) in the following sense. Consider the robustness problem where the class of games of interest is the set of complete information games. Moreover, let this set be defined by requiring an element of the parameter space to have probability one and for this to be commonly known. Formally, consider the set $A$ of types $t^{*}$ in the universal type space $T^{*}$ such that, for some parameter $\theta^{*}$ in the parameter space $\Theta^{*}$, $t_{i}^{*}=\left(1_{\theta^{*}}, 1_{\left(\theta^{*},\left(1_{\theta^{*}}, \ldots, 1_{\theta^{*}}\right)\right)}, \ldots\right)$ for each player $i \in N .{ }^{14}$ Letting $X$ be the universal type

\footnotetext{
${ }^{14}$ For any compact metric space $K$ and any $x \in K, 1_{x}$ is the element in $M(K)$ that assigns
} 
space and $\Psi: X \rightrightarrows S$ be the correspondence of the interim correlated rationalizable actions as in Section 2.2, we can ask: Does $\Psi$ fail to be generically robust on $A$ ? As $A$ is, in this case, meager (nowhere dense in fact) and not dense in $X$, Theorem 1 does not apply and we leave this as an open question. ${ }^{15}$ More generally, this leads to another open question: When $(X, A, S, \Psi)$ is a robustness problem, what can be said, in general, regarding the generic robustness of $\Psi$ on $A$ relative to $X$ when $A$ is meager and not dense in $X$ ?

Additional open questions are: Does Theorem 1 hold when a stronger notion of genericity (say, open and dense as in Corollary 2) is used in the definition of generic robustness? Can a general characterization of the points of discontinuity be given? Can generic robustness results be obtained when the solution correspondence is not upper hemi-continuous?

\section{A Appendix}

\section{A.1 Proof of Theorem 1}

Let $(X, A, S, \Psi)$ be a robustness problem. By Fort's theorem (see Fort (1951, Theorem $2)$ ), there exists a generic set $R$ in $X$ such that $\Psi$ is continuous on $R$.

Before turning to the proof of the first and second parts of the theorem, note that a set is meager if and only if it is contained in a countable union of closed sets each of which has no interior points. Hence, a set is generic if and only if it contains a countable intersection of open dense sets.

\footnotetext{
probability one to $x$.

${ }^{15}$ Under Weinstein and Yildiz's (2007) richness assumption, the question can be reformulated as follows. Let $U=\left\{t \in T^{*}:\left|S^{\infty}(t)\right|=1\right\}$ be the set of type profiles with unique rationalizable action profiles. Then $U$ is open and dense in $X$ by Weinstein and Yildiz (2007, Proposition 2); hence, $U \cap A$ is open in $A$. The question is then whether or not $U \cap A$ is dense in $A$. The denseness of $U \cap A$ in $A$ may fail (I am thankful to an anonymous referee for this argument): Let $\Theta^{*}=[0,1]^{N \times C}$ so that $\theta^{*}$ corresponds to a profile of payoff functions. Consider an open neighborhood of a $\theta^{*}$ corresponding to the payoff function profile of a coordination game. We then obtain a nonempty open subset of $A$ contained in the complement of $U$.
} 
Suppose that $A$ is non-meager in $X$ and suppose, in order to reach a contradiction, that $A \cap R$ is meager in $A$. Then, by Bourbaki (1966, Proposition 2, p. 191) and the comments following Definition 2 in Bourbaki (1966, p. 192), $A \cap R$ is meager in $X$. Since $A \cap R^{c} \subseteq R^{c}$ and $R^{c}$ is meager in $X$, then $A \cap R^{c}$ is meager in $X$. Thus, $A=(A \cap R) \cup\left(A \cap R^{c}\right)$ is meager in $X$, a contradiction. This shows that $A \cap R$ is non-meager in $A$ and established the first part of the theorem.

Suppose next that $A$ is dense in $X$. Since $R$ is generic in $X$, let $\left\{O_{k}\right\}_{k=1}^{\infty}$ be a countable family of open dense sets in $X$ such that $\bigcap_{k=1}^{\infty} O_{k} \subseteq R$. Since $A$ is dense in $X$ and $O_{k}$ is open and dense in $X$, then $A \cap O_{k}$ is dense in $A$ for each $k \in \mathbb{N}$. Indeed, if $x \in A \cap O_{k}^{c}$ and $U$ is an open neighborhood of $x$ in $X$, there exists $y \in O_{k} \cap U$ since $O_{k}$ is dense in $X$ and, as $O_{k} \cap U$ is an open neighborhood of $y$ and $A$ is dense in $X$, there exists $z \in A \cap O_{k} \cap U$. It follows that $\left\{A \cap O_{k}\right\}_{k=1}^{\infty}$ is a countable family of open dense sets in $A$ such that $\bigcap_{k=1}^{\infty}\left(A \cap O_{k}\right) \subseteq A \cap R$, which implies that $A \cap R$ is generic in $A$.

Finally, consider the case where $S$ is finite. Note that $R$ is dense in $X$. Since $\Psi$ is continuous on $R$ and $S$ is finite, then, for each $\tilde{x} \in R$, there exists an open neighborhood $O_{\tilde{x}}$ of $\tilde{x}$ such that $\Psi\left(x^{\prime}\right)=\Psi(\tilde{x})$ for all $x^{\prime} \in O_{\tilde{x}}$. Define $\tilde{O}=\bigcup_{\tilde{x} \in R} O_{\tilde{x}}$ and note that $\tilde{O}$ is open (clearly) and dense (since $R \subseteq \tilde{O}$ and $R$ is dense in $X$ ).

Let $O=A \cap \tilde{O}$. Then $O$ is open in $A$ and, as above, $O$ dense (resp. non-meager) in $A$ if $A$ is dense (resp. non-meager) in $X$. Furthermore, for each $x \in O$, then $x \in O_{\tilde{x}}$ for some $\tilde{x} \in R$ and, hence, $\Psi(x)=\Psi(\tilde{x})=\Psi\left(x^{\prime}\right)$ for all $x^{\prime} \in O_{\tilde{x}}$ and, hence, for all $x^{\prime} \in A \cap O_{\tilde{x}}$. Thus, $U=A \cap O_{\tilde{x}}$ is the desired open neighborhood of $x$ in $A$.

\section{A.2 Proof of Theorem 2}

We start by defining the solution correspondence $\Psi: X \rightrightarrows S$ : For each $U \in X$, let

$$
\Psi(U)= \begin{cases}\{\mu \in \Delta(C): \mu \text { is a correlated equilibrium of } G\} & \text { if } U \in A \\ \{\mu \in \Delta(C): \mu \text { is an equilibrium action distribution of } U\} & \text { otherwise. }\end{cases}
$$

Recall that a correlated equilibrium of $G$ is $\mu \in \Delta(C)$ such that, for each $i \in N$ and $c_{i}, c_{i}^{\prime} \in C_{i}, \sum_{c_{-i} \in C_{-i}} g_{i}\left(c_{i}, c_{-i}\right) \mu\left(c_{i}, c_{-i}\right) \geq \sum_{c_{-i} \in C_{-i}} g_{i}\left(c_{i}^{\prime}, c_{-i}\right) \mu\left(c_{i}, c_{-i}\right)$. 
We next define a topology on $X$ by specifying the neighborhood system $B(U)$ of each point $U$ in $X$ as follows. For each $U \in A^{c}$, let $B(U)=\{B \subseteq X: U \in B\}$, i.e. $B(U)$ coincides with the neighborhood system of $U$ in the discrete topology. ${ }^{16}$ Let $B(0)$ be the neighborhood system of 0 in $[0,1]$, the latter endowed with its usual topology. Then, for each $U \in A$, let $B(U)$ be the collection of subsets $B$ of $X$ such that $\bigcup_{\varepsilon \in O} E(G, \varepsilon) \subseteq B$ for some $O \in B(0)$. Let $\tau$ be the topology generated by this neighborhood system.

We now show that the three properties in the statement of Theorem 2 hold. We start by showing that $\tau$ is a topology on $X$, which follows once we establish the following properties for each $U \in X$ (see Bourbaki (1966)): (a) if $B \in B(U)$ and $B^{\prime} \supseteq B$ then $B^{\prime} \in B(U)$, (b) if $B \in B(U)$ and $B^{\prime} \in B(U)$ then $B \cap B^{\prime} \in B(U)$, (c) $U \in B$ for each $B \in B(U)$, and (d) for each $B \in B(U)$, there exists $B^{\prime} \in B(U)$ such that $B \in B\left(U^{\prime}\right)$ for all $U^{\prime} \in B^{\prime}$. All of these properties are clear when $U \in A^{c}$ and so are (a) and (c) in the case $U \in A$. Thus, assume that $U \in A$.

We next show that (b) holds. Let $B \in B(U)$ and $B^{\prime} \in B(U)$, and let $O_{1}, O_{2} \in B(0)$ be such that $\bigcup_{\varepsilon \in O_{1}} E(G, \varepsilon) \subseteq B$ and $\bigcup_{\varepsilon \in O_{2}} E(G, \varepsilon) \subseteq B^{\prime}$. Then $\bigcup_{\varepsilon \in O_{1} \cap O_{2}} E(G, \varepsilon) \subseteq$ $\left(\bigcup_{\varepsilon \in O_{1}} E(G, \varepsilon)\right) \cap\left(\bigcup_{\varepsilon \in O_{2}} E(G, \varepsilon)\right) \subseteq B \cap B^{\prime}$ and, thus, $B \cap B^{\prime} \in B(U)$. Finally note that (e) holds. Indeed, let $B \in B(U)$ and define $B^{\prime}=B$. Then it follows that $B \in B\left(U^{\prime}\right)$ for each $U^{\prime} \in B^{\prime}$.

The above shows that $X$ is a topological space when endowed with $\tau$. To establish part 1, we first show that $\Psi$ is upper hemicontinuous with nonempty and compact values. That $\Psi$ has nonempty and compact values follows from standard arguments. Note that $X$ is first countable since $\{U\}$ is a countable local base for each $U \in A^{c}$ and, letting $C(0)$ be a countable local base of 0 in $[0,1]$, it follows that $\left\{\bigcup_{\varepsilon \in O} E(G, \varepsilon): O \in C(0)\right\}$ is a countable local base of $U$ for each $U \in A$. We have that $\Psi$ is upper hemicontinuous at $U \in A^{c}$ since $U$ is an isolated point of $X$. Furthermore, for each $U \in A$, since $X$ is first countable, it follows by Kajii and Morris (1997b, Corollary 3.5) that $\Psi$ is upper hemicontinuous at $U$.

\footnotetext{
${ }^{16}$ Thus, we are making each $U \in A^{c}$ be an isolated point in $X$. This choice is made by simplicity since the robustness question being analysed is independent of what happens around a given $U \in A^{c}$.
} 
To complete the proof of part 1 , we now show that $(X, A, S, \Psi)$ reflects Kajii and Morris's (1997b) notion of robustness to incomplete information. Let $U \in A$ be such that $\Psi$ is lower hemicontinuous at $U, \mu$ be a Nash equilibrium action distribution of $G$ and $\delta>0$. Then, for some $V \in B(U), \Psi\left(U^{\prime}\right) \cap B_{\delta}(\mu) \neq \emptyset$ for all $U^{\prime} \in V$, where $B_{\delta}(\mu)$ denotes the ball of radius $\delta$ around $\mu$. Hence, there exists $O \in B(0)$ and, in fact, $\varepsilon^{\prime}>0$ such that $\bigcup_{\varepsilon \in\left[0, \varepsilon^{\prime}\right)} E(G, \varepsilon) \subseteq \bigcup_{\varepsilon \in O} E(G, \varepsilon) \subseteq V$. Letting $\bar{\varepsilon}=\varepsilon^{\prime} / 2>0$, we have that, for all $0 \leq \varepsilon \leq \bar{\varepsilon}$, every $U \in E(G, \varepsilon) \backslash A$ has an equilibrium action distribution $\nu$ such that $\|\mu-\nu\|<\delta$. Furthermore, and clearly, every $U \in A$ has an equilibrium action distribution $\nu$ such that $\|\mu-\nu\|<\delta$, namely $\nu=\mu$. Thus, $\mu$ is robust to incomplete information.

We next prove part 2. It is clear that $A$ is closed since, for each $U \notin A,\{U\} \in$ $B(U)$ and $\{U\} \cap A=\emptyset$. Furthermore, $A$ has an empty interior since each neighborhood of each $U \in A$ contains $E(G, \varepsilon)$ for some $\varepsilon>0$ and, hence, intersects $A^{c}$. Thus, $A$ is nowhere dense and, hence, meager. Furthermore, $A$ is not dense in $X$ since, for each $U \in A^{c},\{U\}$ is an open neighborhood of $U$ and $\{U\} \cap A=\emptyset$.

Finally, we prove part 3. First, note that $A^{c}$ is dense. Indeed, for each $U \in A$ and $B \in B(U)$, let $O \in B(0)$ be such that $U \in \cup_{\varepsilon \in O} E(G, \varepsilon)$ and let $\varepsilon^{\prime}>0$ be such that $\varepsilon^{\prime} \in O$. Then, let $U^{\prime} \in E\left(G, \varepsilon^{\prime}\right)$ and note that $U^{\prime} \in A^{c} \cap B$. Second, to show that $X$ is a Baire space, note that it suffices to show that if a subset $V$ of $X$ is a countable intersection of open dense subsets of $X$, then $V$ is dense in $X$ (see Bourbaki (1966, p. 192)). To this end, let $V_{k}$ be an open dense subset of $X$ for each $k \in \mathbb{N}$ and $V=\bigcap_{k=1}^{\infty} V_{k}$. For each $k \in \mathbb{N}$, we have that $A^{c} \subseteq V_{k}$ since $V_{k}$ is dense and each element of $A^{c}$ is isolated. Hence, $A^{c} \subseteq V$ and, since $A^{c}$ is dense in $X$, so is $V$.

We next prove that $A$ is a Baire space. First note that the relative topology of $A$ is the indiscrete topology. In fact, if $V$ is a nonempty open subset of $A$, then $V=A \cap V^{\prime}$ for some open $V^{\prime}$ in $X$. Since $V$ is nonempty, then $V^{\prime} \in B(U)$ for some $U \in A$ and, hence, $\bigcup_{\varepsilon \in O} E(G, \varepsilon) \subseteq V^{\prime}$ for some $O \in B(0)$. Since $A \subseteq \bigcup_{\varepsilon \in O} E(G, \varepsilon)$, then $A \cap V^{\prime}=A$, i.e. $V=A$. Since the relative topology of $A$ is the indiscrete topology, it follows that $A$ is the unique nonempty open set in $A$, that the empty set is the unique nowhere dense set in $A$, that $A$ is non-meager and, hence, that $A$ is a 
Baire space. This concludes the proof.

\section{A.3 Proof of Remark 1}

Let $(X, A, S, \Psi)$ and $\Phi$ be as in the statement of the remark. Let $R$ be residual in $X$ and such that $\Psi$ is continuous on $R$. Since $A$ is closed, we have that $R^{c}$ is meager both in $A^{c}$ and in $X$ (see Bourbaki (1966, p. 192) for the former). Thus, $R \cap A^{c}$ is residual both in $A^{c}$ and in $X$. As $\Psi$ is continuous on $R \cap A^{c}$ and $\Phi(x)=\Psi(x)$ for each $x \in A^{c}$, it follows that $\Phi$ is generically robust on $X$ and on $A^{c}$, both relative to $X$.

\section{References}

Bourbaki, N. (1966): Elements of Mathematics, General Topology, vol. 2. AddisonWesley, London.

Brandenburger, A., and E. Dekel (1993): "Hierarchies of Beliefs and Common Knowledge," Journal of Economic Theory, 59, 189-198.

Carbonell-Nicolau, O. (2010): "Essential Equilibria in Normal-Form Games," Journal of Economic Theory, 145, 421-431.

Carlsson, H., and E. van Damme (1993): "Global Games and Equilibrium Selection," Econometrica, 61, 989-1018.

Carmona, G. (2013): Existence and Stability of Nash Equilibrium. World Scientific, Singapore.

Chen, Y.-C. (2012): "A Structure Theorem for Rationalizability in the Normal Form of Dynamic Games," Games and Economic Behavior, 75, 587-597.

Chen, Y.-C., S. Takahashi, and S. Xiong (2014): "The Weinstein-Yildiz Selection and Robust Predictions with Arbitrary Payoff Uncertainty," National University of Singapore and University of Bristol. 
Chen, Y.-C., And S. Xiong (2013): "Genericity and Robustness of Full Surplus Extraction," Econometrica, 81, 825-847.

Ely, J., And M. PȨSKi (2011): "Critical Types," Review of Economic Studies, 78, 907-937.

Fort, M. K. (1951): "Points of Continuity of Semi-Continuous Functions," Publicationes Mathematicae Debrecen, 2, 100-102.

Fremlin, D. H. (2003): Measure Theory, vol. 4: Topological Measure Spaces. Torres Fremlin, Colchester.

KaJII, A., AND S. MorRIS (1997a): "Refinements and Higher Order Beliefs: A Unified Survey," mimeo.

_ (1997b): "The Robustness of Equilibria to Incomplete Information," Econometrica, 65, 1283-1309.

Kelley, J. (1955): General Topology. Springer, New York.

Lipman, B. (2003): "Finite Order Implications of Common Priors," Econometrica, $71,1255-1267$.

Mertens, J.-F., And S. Zamir (1985): "Formulation of Bayesian Analysis for Games with Incomplete Information," International Journal of Game Theory, 14, $1-29$.

Penta, A. (2012): "Higher Order Uncertainty and Information: Static and Dynamic Games," Econometrica, 80, 631-660.

— (2013): "On the Structure of Rationalizability for Arbritary Spaces of Uncertainty," Theoretical Economics, 8, 405-430.

Scalzo, V. (2013): "Essential Equilibria of Discontinuous Games," Economic Theory, 54, 27-44. 
Weinstein, J., AND M. YiLdiz (2007): "A Structure Theorem for Rationalizability with Application to Robust Predictions of Refinements," Econometrica, 75, 365400.

(2017): "Interim Correlated Rationalizability in Infinite Games," Journal of Mathematical Economics, 72, 82-87. 